\title{
A Dosimetric Comparison of Double Arc Volumetric Modulated Arc Therapy with Large Field Intensity Modulated Radiation Therapy for Head and Neck Cancer
}

\author{
Jayapalan Krishnan ${ }^{*}$, Suresh Rao1, Sanath Hegde'1, Jayarama Shetty², Shambhavi1 \\ ${ }^{1}$ Department of Radiation Oncology, Mangalore Institute of Oncology, Mangalore, India \\ ${ }^{2}$ Department of Radiation Oncology, K. S. Hegde Medical Academy, Mangalore, India \\ Email: "nkjayapalceg37@gmail.com
}

Received 23 October 2015; accepted 15 November 2015; published 18 November 2015

Copyright (C) 2015 by authors and Scientific Research Publishing Inc.

This work is licensed under the Creative Commons Attribution International License (CC BY). http://creativecommons.org/licenses/by/4.0/

(c) (i) Open Access

\section{Abstract}

Background: This Study Evaluate two state of Art techniques, Rapid Arc and large-field sliding window IMRT in terms of dosimetric end points and delivery time for head and neck cancer. Materials and Methods: 22 patients with head and neck cancer were selected for a planning comparative study. All patients went to CT-simulation in supine position. PTVs were delineated for two dose level of prescription $70 \mathrm{~Gy}$ to the boost-PTV ${ }_{70 \mathrm{~Gy}}$ and $54 \mathrm{~Gy}$ to the elective-PTV ${ }_{54 \mathrm{~Gy}}$ in 35 equal fraction/day. Simultaneous Integrated Boost (SIB) technique plan was generated for all patients and optimized with both techniques, Rapid Arc and IMRT with similar planning objectives. Dose of all plans of both techniques were calculated for $6 \mathrm{MV}$ photon using AAA implemented in Eclipse treatment planning system (10.0.39) with calculating grid size of $2.5 \mathrm{~mm}$. Results: Comparison of Rapid Arc and IMRT plans in every patient showed significantly higher conformity index $\left(\mathrm{CI}_{95 \%}\right)(p$ $=0.021)$ and sparing of the all OARs with Rapid Arc. The average homogeneity Index (Hi95\%) of lower prescribed dose target PTV $_{54 \mathrm{~Gy}}$ which is in proximity to Higher dose prescribed target PTV $_{70 \mathrm{~Gy}}$ was improved significantly with rapid Arc $(p=0.0001)$. $\mathrm{D}_{1 \%}$ of spinal cord dose reduced significantly $(p=0.047)$ with Rapid Arc and the average mean dose of both left-parotid (21.26 $\pm 8.5 \mathrm{~Gy})$, right-parotid $(22.37 \pm 7.44 \mathrm{~Gy})$ were received lesser than with IMRT $(22.78 \pm 11.2 \mathrm{~Gy}$ and $24.1 \pm$ $7.96 \mathrm{~Gy})$ respectively. A significantly less monitor unit (MU) was required to deliver the plan ( $p<$ $0.00001)$ with significantly lesser treatment time $(p<0.00001)$. Conclusion: Rapid Arc technique was superior to IMRT in sparing the OARs without compromising target coverage and delivered the plan with lesser monitor unit and treatment time.

\footnotetext{
*Corresponding author.
}

How to cite this paper: Krishnan, J., et al. (2015) A Dosimetric Comparison of Double Arc Volumetric Modulated Arc Therapy with Large Field Intensity Modulated Radiation Therapy for Head and Neck Cancer. International Journal of Medical Physics, Clinical Engineering and Radiation Oncology, 4, 353-363. http://dx.doi.org/10.4236/ijmpcero.2015.44042 
Keywords

Head and Neck, Rapid Arc, VMAT, IMRT, Planning Study

\section{Introduction}

Radiotherapy techniques have evolved into an extremely complex process with new imaging modalities, delivery systems and patient immobilization devices. These technological advances have made it possible to reduce the dose to normal tissue structures and consequently minimize the risk of toxicity and morbidity, while allowing for dose escalation to the tumor volumes, potentially leading to improved loco-regional control [1]. Head and neck cancer is histologically heterogeneous and organs at risk have less tolerance to radiation. Due to complex shape of target and close proximity of critical structure, the most common method of head and neck cancer radiotherapy treatment is intensity modulated radiotherapy (IMRT). These techniques address the drawbacks of conventional 3DCRT techniques, such as under-dosage of target regions and excessive radiation to surrounding normal structures [2]. An advantage of IMRT technology over 3DCRT for the treatment of nasopharyngeal carcinoma is improved dose distribution within the target area, better dose hotspot control, and reduced radiation exposure to organs at risk (OAR), including the parotids, brain stem, spinal cord and oral cavity [3]-[5].

Large field IMRT (LF-IMRT), is a standard IMRT plan which slightly differs in delivery method. For large field delivery, due to the limitation of mlc travelling length, the standard sliding window IMRT plan can create sub-fields and deliver dynamically. In the standard IMRT, mlc carriage moves to next position to cover the subfields. Each one of these sub-fields require mode up separately to deliver. LF-IMRT does not require to mode up each sub-field separately and the all subfields in a large field does mode up collectively. While moving the mlc carriage to deliver the subsequent subfields dynamically, the dose rate falls to zero to reach to the planned position and continue to deliver the plan. These slightly reduce the overall treatment time.

LF-IMRT plan, is similar to standard IMRT plan and requires multiple fixed-angle radiation beams with many treatment segments or sub-fields, leading to lengthy treatment delivery times. Extended time on the table reduces comfort of patient and increases intra-fraction motion of the tumor or organs at risk (OARs). Therefore, the availability of new technologies is of great interest.

Volumetric modulated arc therapy, based on the original investigation of Otto [6] has been introduced in clinical practice in several institutes after an intensive validation at the planning level, compared with intensity modulated radio therapy (IMRT) or other approaches. Rapid Arc, the Varian medical systems (palo alto, CA) solution of VMAT, is implemented as the progressive Resolution Optimization algorithm-III in the Eclipse treatment planning system (varian medical system). The optimization process is based on an iterative inverse planning process aiming to simultaneously optimize the instantaneous multi leaf collimator (mlc) positions, dose rate, and gantry rotation speed to achieve the desired dose distribution.

Recent studies have examined dosimetric difference between IMRT and VMAT for various cancers, with the consensus being that VMAT produces a similar or superior plan to IMRT with a more efficient delivery [7]. Therefore, the aim of this study was to compare the dosimetric and technical aspects of VMAT and LF-IMRT for the radiotherapy treatment of head and neck cancer.

\section{Methods and Materials}

\subsection{Patient Selection}

Twenty two patients presenting head and neck tumors, were considered for VMAT. Table 1 shows the descriptive data of the patients. It includes 19 males and 3 females with a median age of 62 years (range 40 - 76 years). This study was approved by the ethics committee and informed consent for treatment was obtained from all the patients. To have unbiased comparison of VMAT and IMRT plan quality, patients who were treated with VMAT were also planned IMRT. Each patient served as his own control for the dosimetric and technical parameters comparison.

\subsection{Volume Definition and Treatment Planning}

All patients were simulated in supine position using a thermoplastic mask and shoulder retraction. A planning 
Table 1. Summary of patients characteristics at treatment start.

\begin{tabular}{|c|c|c|}
\hline Number of Patients & & 22 \\
\hline \multirow[t]{8}{*}{ Site } & PFS & 8 \\
\hline & Post Cricoid & 2 \\
\hline & Base of Tongue & 2 \\
\hline & Tongue & 3 \\
\hline & Supraglotties & 2 \\
\hline & Hypopharynx & 2 \\
\hline & Oropharynx & 1 \\
\hline & Epiglotties & 2 \\
\hline \multirow[t]{2}{*}{ Sex } & Males & 19 \\
\hline & Females & 3 \\
\hline Age (Years) & Median (Range) & $62(40.76)$ \\
\hline \multirow[t]{3}{*}{ Stage } & II & 5 \\
\hline & III & 10 \\
\hline & IV & 7 \\
\hline \multirow[t]{3}{*}{ Chemotherapy } & No ChT & 4 \\
\hline & CisPl & 16 \\
\hline & CarboPl & 2 \\
\hline \multicolumn{3}{|l|}{ Radiation Dose } \\
\hline Prescription & SIB, 70/54 Gy in 33 Fraction & 22 \\
\hline
\end{tabular}

SIB: Simultaneous Integrated Boost; PFS: Pyriform Fossa Sinus; ChT: Chemotherapy; CisPl: Cisplatin; CarboPl: Carboplatin.

CT scan with contrast was performed with slice thickness of 3mm. A planning MRI scan was performed in the same position. The two images were transferred to the Eclipse planning systems (10.0.39) and were fused using rigid registration algorithm for delineation of target and organs at risk (OARs). Gross Tumour Volume (GTV) was defined as the gross extent of tumour shown by imaging, including all involved (positive) lymph nodes. MRI and in few cases FDG-PET were used in the delineation of GTV. On the basis of the primary tumour size and involved nodes, the $\mathrm{CTV}_{70}$ was defined as GTV (guided by clinical criteria and FDGPET imaging whenever available) plus a margin for microscopic spread, and the $\mathrm{CTV}_{54}$ included precautionarily uninvolved nodes defined as CTV. Planning Target Volume (PTV) was generated by expanding the CTV by 5 mm in all directions [ICRU 50 and 62]. Organs at risks (OARs) such as Spinal Cord, left and right Parotids Larynx and uninvolved Oral Cavity were contoured. In addition, the healthy tissue was defined as the patient's volume included in the CT data set and excluded all PTV volumes.

Dose was prescribed to mean PTV dose for the high dose level as simultaneous integrated boost (SIB) with two dose levels of $54 \mathrm{~Gy}$ and $70 \mathrm{~Gy}$ in 35 fractions (1.54 and 2.0 Gy/fraction, respectively). All patients were treated with $6 \mathrm{MV}$ X-ray beams delivered by varian UNIQUE performance equipped with a millennium multi leaf collimator (mlc) with 120 leaves (spatial resolution of $5 \mathrm{~mm}$ at isocentre for the central $20 \mathrm{~cm}$ and of $10 \mathrm{~mm}$ in the outer $2 \times 10 \mathrm{~cm}$, leaf transmission of 1.86\%). For each patient two plans were prepared with Similar Plan objectives, one using large fields sliding window IMRT and another using Rapid Arc. The sliding window IMRT plan consisted on 7 - 9 gantry angles. For most of the patients gantry angle was fixed at $0^{\circ}, 40^{\circ}, 80^{\circ}, 120^{\circ}$, $160^{\circ}, 200^{\circ}, 240^{\circ}, 280^{\circ}$ and $320^{\circ}$. Beam angles were selected in order to avoid opposite entrance. All beams were coplanar and collimator angle set to $20^{\circ}$ to reduce the tongue and groove effect. Plan optimization was performed on the basis of dose-volume constraints with fixed $400 \mathrm{MU} / \mathrm{min}$. The VMAT consisted on two arcs 
(clockwise and counter clockwise direction) of $358^{\circ}$ each and collimator angles set to $30^{\circ}$ and $330^{\circ}$ were used. Both arcs were coplanar. VMAT plan optimization with PRO-III implemented in Eclipse planning system was performed on the basis of appropriate dose volume constraints which were used for IMRT optimization. A maximum dose rate of $600 \mathrm{MU} / \mathrm{min}$ was applied. Dose of both the plans were calculated using anisotropic analytical algorithm (AAA) with calculating grid size of $2.5 \mathrm{~mm}$.

For both Rapid Arc and LF-IMRT plans, dose volume objectives for PTVs were: At least 95\% of the PTV coverage is $95 \%$ of the prescribed dose and dose maximum not to exceed $107 \%$ of the prescribed dose. Concerning OARs, the constraints were following: Spinal cord: $\mathrm{D}_{1 \%}<46 \mathrm{~Gy}$; Parotids (left and right analysed separately): $\mathrm{D}_{\text {mean }}<26 \mathrm{~Gy}, \mathrm{~V}_{30 \mathrm{~Gy}}<45 \%$; larynx: $\mathrm{D}_{\text {mean }}<45 \mathrm{~Gy}$ and uninvolved oral cavity: $\mathrm{D}_{\text {mean }}<30 \mathrm{~Gy}$. For healthy tissue (defined as the entire body volume included in the CT scan subtracted all PTVs), various thresholds like $\mathrm{V}_{5 \mathrm{~Gy}}, \mathrm{~V}_{30 \mathrm{~Gy}}$ and mean dose were reduced to as low as possible.

\subsection{Evaluation Parameter}

Evaluation of plans was based on dose volume histogram (DVH) analysis. For each PTV, the values of $\mathrm{D}_{98 \%}$ and $\mathrm{D}_{2 \%}$ (Dose received by the $98 \%$ and $2 \%$ of the target volume) were defined as metrics for minimum and maximum doses respectively. Also $\mathrm{D}_{80 \%}, \mathrm{D}_{50 \%}$ and $\mathrm{V}_{107 \%}$ were reported. The homogeneity of the dose distribution was measured by $D_{5 \%}-D_{95 \%}$ and expressed as $H I=\left(D_{5 \%}-D_{95 \%}\right) / D_{50 \%}$. The lower HI value, the better dose homogeneity. Ideal value is equal to zero. Conformity of Dose was scored as conformity index $\left(\mathrm{CI}_{95 \%}\right)$ which was computed for each plan using the equation $\mathrm{CI}_{95 \%}=\left(\mathrm{TV}_{\mathrm{PI}} / \mathrm{TV}\right) \times\left(\mathrm{TV}_{\mathrm{PI}} / \mathrm{PI}\right)$. Where $\mathrm{TV}_{\mathrm{PI}}$ represents the target volume with in the $95 \%$ of prescribed iso-dose volume, TV is the volume of PTV and PI is the volume of $95 \%$ of prescribed iso-dose volume [8]. A perfect plan would have a $\mathrm{TV}_{\mathrm{PI}}=\mathrm{TV}=\mathrm{PI}$ then, $\mathrm{CI}_{95 \%}=1$.

In OARs, the mean dose, the maximum dose $\left(\mathrm{D}_{1 \%}\right)$ and appropriate values of $\mathrm{V}_{\mathrm{xGy}}$ (Volume receiving at least $\times$ Gy) were analysed. For healthy tissue, the integral dose of the absorbed dose extended to over all voxels excluding the volume of all targets. It was computed for each plan using the equation, Integral Dose $={ }_{i=1} \Sigma^{\mathrm{m}} \mathrm{V}_{\mathrm{i}} \mathrm{D}_{\mathrm{i}}$. Where $\mathrm{V}_{\mathrm{i}}$ and $\mathrm{D}_{\mathrm{i}}$ are volume and dose of voxel "i”.

Technical features of treatment have been reported in terms of main delivery parameters: total monitor unit (MU) per fraction and treatment time (defined as Beam on time plus machine programming time and excluding patient positioning and imaging procedures). Results of pre treatment plan quality assurance were reported as gamma agreement Index(GAI) (i.e., percentage of modulated field area passing the $\gamma$-index criteria of Low et al. [9], with thresholds on dose difference set to $\Delta D=3 \%$ of the significant maximum dose and distance to agreement set to DTA $=3 \mathrm{~mm}$ ). Measurements and analysis were performed by means of the GlAaS methodology described in [10] [11] based on absorbed dose to water derived from EPID measurements. Pre treatment dosimetry was considered satisfactory if gamma agreement index (GAI) $\geq 95 \%$.

Statistical analysis was performed using SPSS (version 16.0.0, SPSS, Chicago, USA). Independent student " $\mathrm{t}$ " test was used to compare both treatment techniques. A $p$ value $<0.05$ was considered statistically significant.

\section{Results}

Figure 1 shows the axial, sagittal and coronal view of dose distribution and refers to one patient. Figure 2 and Figure 3 show the comparison DVH for PTVs and OARs respectively. Table 2 and Table 3 give an overview of the numerical results from average DVH analysis on PTVs and OARs respectively and report mean values \pm standard deviation (SD) and " $p$ " values to assess the relative inter-patient variability. Table 4 gives details of the technical characteristics.

As it is now, it is difficult to understand, the target volume was to receive at least $95 \%$ of the prescribed dose, but has received higher than $96 \%$ of the target volumes in both techniques. Mean dose of PTV 70 Gy was achieved better (70.56 $\pm 1.10 \mathrm{~Gy})$ with Rapid Arc without increasing dose maximum than (71.66 $\pm 1.08 \mathrm{~Gy})$ with IMRT. Rapid Arc plans achieved higher dose conformity index $\left(\mathrm{CI}_{95 \%}\right)(0.906 \pm 0.029$, closer to a value of 1$)$ than with IMRT $(0.868 \pm 0.067)$ and the $p=0.021$ shows significant difference. The Homogeneity index $\left(\mathrm{HI}_{95 \%}\right)$ statistically does not differ in both techniques but, mean homogeneity index $\left(\mathrm{HI}_{95 \%}\right)$ shows higher with Rapid Arc $(0.089 \pm 0.020)$ than with IMRT $(0.097 \pm 0.03)$. For PTV $54 \mathrm{~Gy}$ the coverage was adequate in both techniques. However, homogeneity index was achieved better with Rapid Arc (0.097 \pm 0.3$)$ than with IMRT (0.25 \pm 0.10$)$.

Spinal Cord dose was below the tolerance limit for both techniques. However, $\mathrm{D}_{1 \%}$ of Spinal cord with Rapid Arc was $37.7 \pm 4.67 \mathrm{~Gy}$ and with IMRT was $40.68 \pm 7.14 \mathrm{~Gy}$. Both left parotid and right parotid received with 


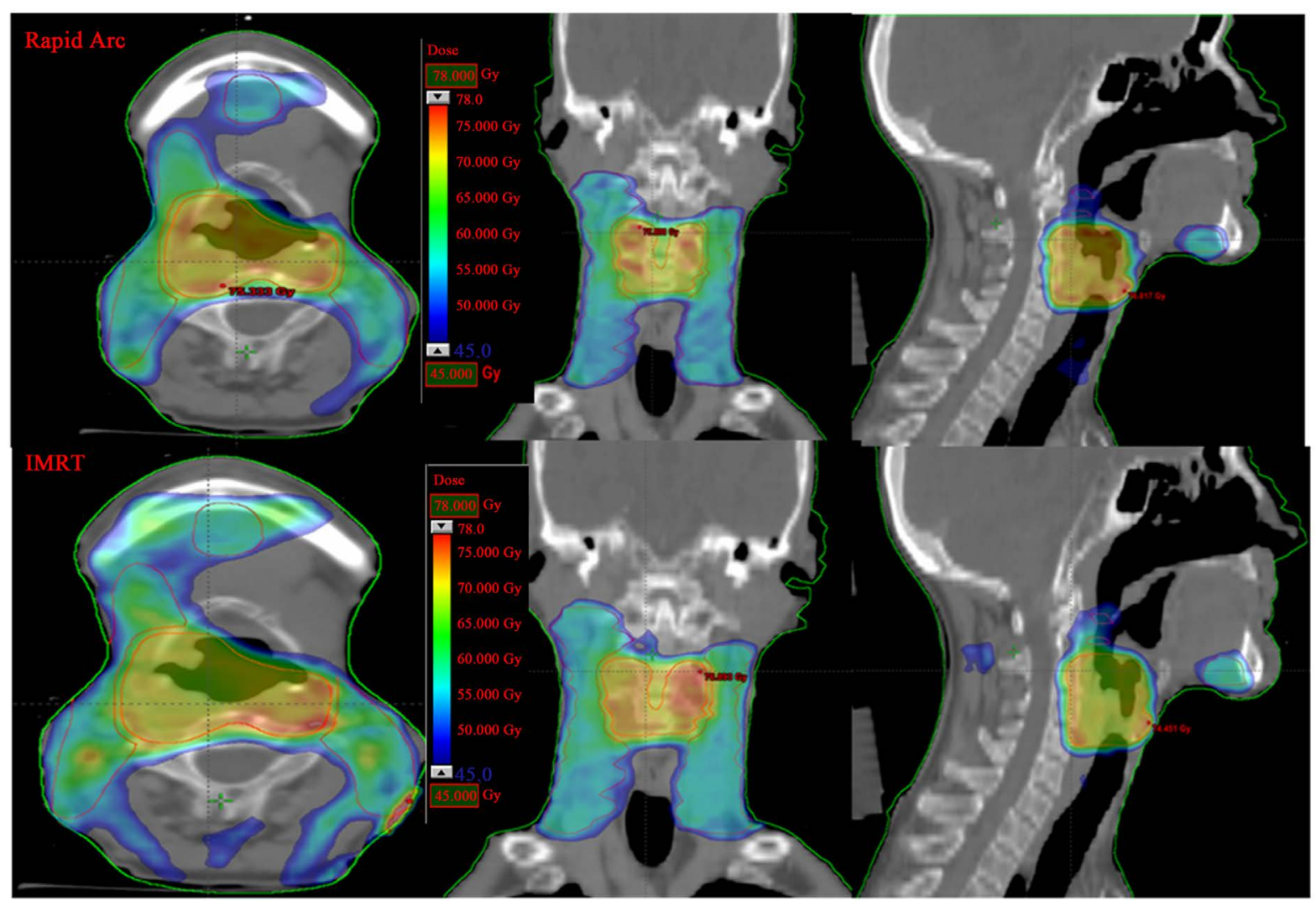

Figure 1. Dose distribution for one patient under study for axial, coronal and sagittal views.

Table 2. Summary of dosimetric results for the $\mathrm{PTV}_{70 \mathrm{~Gy}}$ and $\mathrm{PTV} \mathrm{V}_{54 \mathrm{~Gy}}$.

\begin{tabular}{|c|c|c|c|c|c|c|}
\hline Parameters & & Objective & $\begin{array}{l}\text { Rapid Arc } \\
\text { Mean } \pm \text { SD }\end{array}$ & $\begin{array}{l}\text { LF-IMRT } \\
\text { Mean } \pm \text { SD }\end{array}$ & $\begin{array}{c}p \text {-Value } \\
\text { (Independent Student } \mathrm{t} \text { test) }\end{array}$ & $\begin{array}{l}\text { Rapid Arc vs } \\
\text { LF-IMRT }\end{array}$ \\
\hline \multirow[t]{8}{*}{$\mathrm{PTV}_{70 \mathrm{~Gy}}$} & Mean (Gy) & 70 Gy & $70.56 \pm 1.1$ & $71.66 \pm 1.08$ & 0.011 & $\mathrm{RA}>\mathrm{IMRT}$ \\
\hline & D2\% (Gy) & $<74.9$ Gy & $74.32 \pm 1.5$ & $74.06 \pm 1.9$ & NS & - \\
\hline & D98\% (Gy) & $>66.5$ Gy & $67.95 \pm 1.2$ & $67.22 \pm 1.4$ & NS & - \\
\hline & D50\% (Gy) & 70 Gy & $71.7 \pm 1.1$ & $70.7 \pm 1.0$ & 0.003 & $\mathrm{RA}>\mathrm{IMRT}$ \\
\hline & D80\% (Gy) & 70 Gy & $70.52 \pm 1.1$ & $69.45 \pm 1$ & 0.002 & RA $>$ IMRT \\
\hline & V107 (\%) & $<2 \%$ & $2.1 \pm 1.2$ & $2.9 \pm 1.8$ & NS & - \\
\hline & HI95\% & 0 & $0.089 \pm 0.02$ & $0.097 \pm 0.03$ & NS & - \\
\hline & CI95\% & 1 & $0.906 \pm 0.03$ & $0.868 \pm 0.07$ & 0.021 & $\mathrm{RA}>\mathrm{IMRT}$ \\
\hline \multirow[t]{4}{*}{$\mathrm{PTV}_{54 \mathrm{~Gy}}$} & D2\% (Gy) & $<57.78 \mathrm{~Gy}$ & $63.5 \pm 3.3$ & $64.1 \pm 5.3$ & NS & - \\
\hline & D98\% (Gy) & $>51.3$ Gy & $50.4 \pm 2.19$ & $50.3 \pm 2.1$ & NS & - \\
\hline & D50\% (Gy) & 50 Gy & $56.1 \pm 1.5$ & $55.68 \pm 1.9$ & NS & - \\
\hline & HI95\% & 0 & $0.097 \pm 0.3$ & $0.25 \pm 0.10$ & $<0.0001$ & $\mathrm{RA}>\mathrm{IMRT}$ \\
\hline
\end{tabular}

$\mathrm{Dx} \%=$ dose received by the $\mathrm{X} \%$ of the volume; $\mathrm{Vx} \%=$ Volume receiving at least $\mathrm{X} \%$ of the prescribed dose; $\mathrm{CI}=$ Conformity Index; $\mathrm{HI}=\mathrm{Homo}-$ geneity Index. 


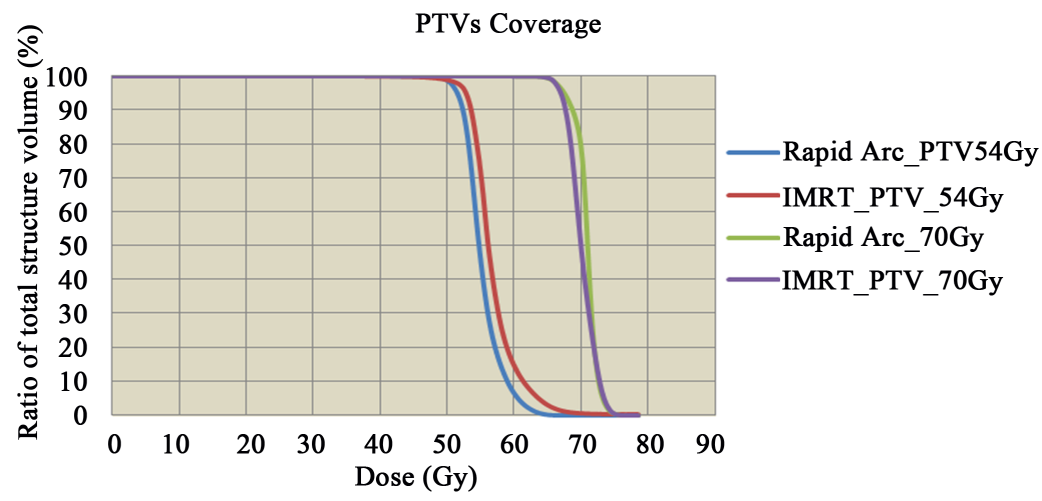

Figure 2. DVH for PTV of a patient's plans. HI95\% of PTV ${ }_{54 \text { Gv }}$ shows significantly better with Rapid Arc and no significant changes in PTV_70 Gy.

Table 3. Summary of dosimetric results for organ at risk and healthy tissue.

\begin{tabular}{|c|c|c|c|c|c|c|}
\hline Organ & & Objective & $\begin{array}{l}\text { Rapid Arc } \\
\text { Mean } \pm \text { SD }\end{array}$ & $\begin{array}{l}\text { LF-IMRT } \\
\text { Mean } \pm \text { SD }\end{array}$ & $\begin{array}{c}p \text {-Value } \\
\text { (Independent Student t test) }\end{array}$ & $\begin{array}{l}\text { Rapid Arc vs } \\
\text { LF-IMRT }\end{array}$ \\
\hline Spinal Cord & D1\% & $<46 \mathrm{~Gy}$ & $37.7 \pm 4.7$ & $40.7 \pm 7.1$ & 0.047 & $\mathrm{RA}<\mathrm{IMRT}$ \\
\hline \multirow[t]{2}{*}{ Lt.Parotid } & Mean (Gy) & $<26 \mathrm{~Gy}$ & $21.26 \pm 8.5$ & $22.78 \pm 11.2$ & NS & - \\
\hline & V30Gy (\%) & $<45 \%$ & $22.37 \pm 14.6$ & $22.49 \pm 19.6$ & NS & - \\
\hline \multirow[t]{2}{*}{ Rt.Parotid } & Mean (Gy) & $<26 \mathrm{~Gy}$ & $22.37 \pm 7.43$ & $24.1 \pm 8.0$ & NS & - \\
\hline & V30Gy (\%) & $<45 \%$ & $25.52 \pm 13.43$ & $26.7 \pm 14.1$ & NS & - \\
\hline Larynx & Mean (Gy) & $<45 \mathrm{~Gy}$ & $37.49 \pm 2.7$ & $41.7 \pm 4.2$ & 0.002 & $\mathrm{RA}<\mathrm{IMRT}$ \\
\hline Oral cavity & Mean (Gy) & $<30 \mathrm{~Gy}$ & $28.22 \pm 4.3$ & $32.7 \pm 3.8$ & 0.004 & \\
\hline Healthy & Mean (Gy) & Minimise & $9.02 \pm 2.9$ & $10.3 \pm 3.3$ & NS & - \\
\hline \multirow[t]{5}{*}{ Tissue } & V5 Gy (\%) & Minimise & $37.9 \pm 9.3$ & $37.0 \pm 9.7$ & NS & - \\
\hline & V30 Gy (\%) & Minimise & $10.1 \pm 3.9$ & $13.1 \pm 5.2$ & 0.037 & $\mathrm{RA}<\mathrm{IMRT}$ \\
\hline & Integral & & & & & \\
\hline & Dose & & & & & \\
\hline & $10^{5} \mathrm{~cm}^{3} \cdot \mathrm{Gy}$ & Minimise & $1.147 \pm 0.705$ & $1.489 \pm 0.758$ & NS & - \\
\hline
\end{tabular}

$\mathrm{Dx} \%$ = dose received by the $\mathrm{X} \%$ of the volume; $\mathrm{Vx} \%=$ Volume receiving at least $\mathrm{X} \%$ of the prescribed dose.

Table 4. Summary of technical characteristics of plans.

\begin{tabular}{|c|c|c|c|c|c|}
\hline Parameters & Objective & $\begin{array}{l}\text { Rapid Arc } \\
\text { Mean } \pm \text { SD }\end{array}$ & $\begin{array}{l}\text { LF-IMRT } \\
\text { Mean } \pm \text { SD }\end{array}$ & $\begin{array}{c}p \text {-Value } \\
\text { (Independent Student } t \text { test) }\end{array}$ & $\begin{array}{l}\text { Rapid Arc vs } \\
\text { LF-IMRT }\end{array}$ \\
\hline \multicolumn{6}{|l|}{ Treatment } \\
\hline Time (min) & & $2.14 \pm 0.43$ & $9.16 \pm 2.5$ & $<0.00001$ & RA $<$ IMRT \\
\hline \multicolumn{6}{|l|}{ Monitor } \\
\hline Unit & & $523.1 \pm 116$ & $2127 \pm 570$ & $<0.00001$ & RA $<$ IMRT \\
\hline GAI 3\%, 3 mm (\%) & $>95 \%$ & $97.84 \pm 1.1$ & $95.23 \pm 1.7$ & - & - \\
\hline
\end{tabular}

GAI = Gamma Agreement Index. 

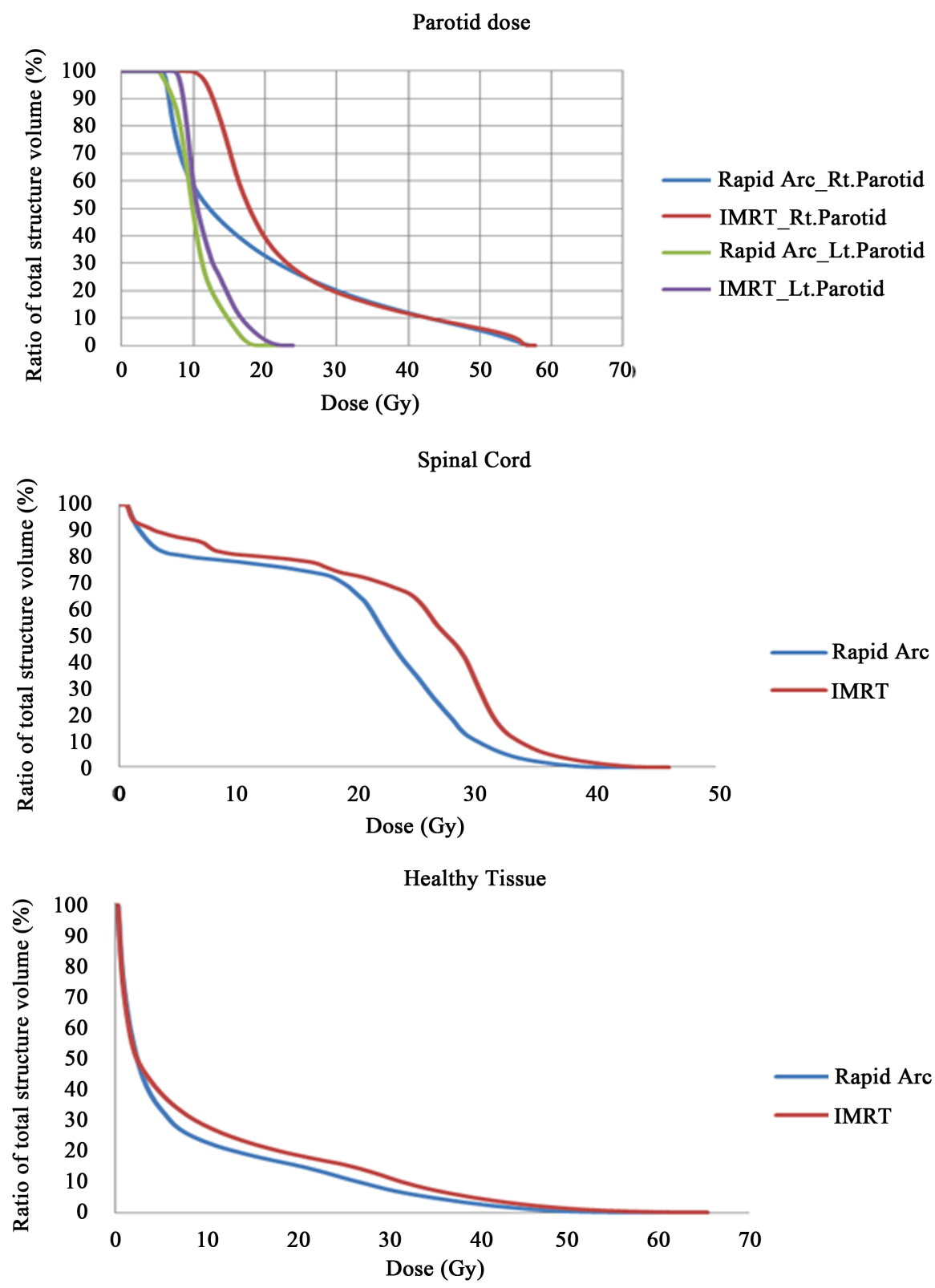

Figure 3. Average DVH for OARs of a patient's plans. Parotids, Spinal cord were spared significantly higher with Rapid arc than IMRT. Irradiated healthy tissue volume by higher dose was lesser with Rapid Arc and no significant difference found in the low dose irradiation volume.

VMAT are $21.26 \pm 8.5 \mathrm{~Gy}$ and $22.37 \pm 7.44$ Gy which are lesser than with IMRT $22.78 \pm 11.2$ Gy and $24.1 \pm$ 7.96 Gy respectively. Average mean dose Larynx was (37.49 \pm 2.7 Gy) with Rapid Arc than with IMRT (41.7 \pm $4.2 \mathrm{~Gy}$ ). Likewise, the average mean dose of uninvolved oral cavity also was lesser with rapid Arc. Concerning Healthy Tissue, Lower dose irradiation volume $\mathrm{V}_{5 \mathrm{G} y}$ was little higher with Rapid Arc $(37.90 \% \pm 9.33 \%)$ than with IMRT $(37 \% \pm 9.67 \%)$. However, higher dose irradiation volume $\mathrm{V}_{30 \mathrm{~Gy}}$ was lesser with Rapid $\operatorname{Arc}(10.07 \%$ $\pm 3.93 \%)$ than with IMRT $(13.1 \% \pm 5.2 \%)$. The Integral dose was with Rapid Arc $\left(1.1475 \times 10^{5} \pm 0.705 \times 10^{5}\right.$ $\left.\mathrm{cm}^{3} \cdot \mathrm{Gy}\right)$ and with IMRT $\left(1.489 \times 10^{5} \pm 0.778 \times 10^{5} \mathrm{~cm}^{3} \cdot \mathrm{Gy}\right)$.

The average monitor unit(MU) needed to deliver the dose of $200 \mathrm{cGy} /$ fraction was $523 \pm 116 \mathrm{MU}$ for Rapid Arc and $2127 \pm 570$ for the 9 field IMRT plan $(p<0.00001)$. The total Beam On time (Excluding patient setup and Image verification) was $2.14 \pm 0.43 \mathrm{~min}$ for Rapid Arc and $9.16 \pm 2.5 \mathrm{~min}$ for IMRT $(p<0.00001)$. Pre- 
treatment quality assurance of all plans resulted in average gamma agreement index (GAI). The GAI scored with $3 \mathrm{~mm}$ and $3 \%$ constraints was $97.84 \% \pm 1.1 \%$ with Rapid Arc plans and $95.23 \% \pm 1.7 \%$ with IMRT plans. The acceptable GAI threshold was set to $95 \%$ as a reference in the hospital.

\section{Discussion}

Different planning studies comparing the volumetric modulated arc therapy with conventional IMRT in different sites have been published [12]-[15]. The study published by Richard et al. [16]. Shows VMAT had shorter Beam-On time and more homogeneous dose distribution compared with 7 field, step and shoot IMRT for prostate cancer. In another study published by Mahantshetty, swamidas Jamema et al. [17] shows that Rapid Arc allows the generation of adequate dose distribution with high target homogeneity, sufficient sparing of organs at risk and minimization of patient moment compared to IMRT. The mean dose of PTV $\mathrm{F}_{70 \mathrm{~Gy}}$ received adequately with both techniques, however, Rapid Arc mean dose was differ significantly $(p=0.011)$ than IMRT. Conformity Index $\left(\mathrm{CI}_{95 \%}\right)(p=0.021)$, dose of $\mathrm{D}_{80 \%}(p=0.002)$ and $\mathrm{D}_{50 \%}(p=0.003)$ were significantly higher with Rapid Arc than IMRT, while the $\mathrm{V}_{107 \%}(p=0.288)$ and homogeneity Index $\left(\mathrm{HI}_{95 \%}\right)(p=0.28)$ did not differ significantly with IMRT. Therefore, the dose fall-off with Rapid Arc is higher than IMRT. The advantage of steep dose fall-off with Rapid Arc technique achieved higher Homogeneity for proximity target with low dose prescription PTV 54 Gy significantly $(p=0.0001)$ than IMRT.

When using IMRT for irradiation of oropharyangeal cancer salivary functions was less impaired, but the majority of the patients still suffered from some degree of xerostomia [18]-[20]. Braam et al. showed--that the normal tissue complication probability (NTCP) at several time points after radiation therapy was less than $20 \%$ only if the mean dose to the parotid glands was lower than 25 Gy [21]. The average mean dose of both left parotid and right parotid received with VMAT were lesser than with IMRT. However, significantly not differ. $V_{30 G y}$ of parotid was also lesser with Rapid Arc than IMRT, It ensures that irradiation of higher dose volume is reduced.

Kirk Patrice et al. [22]. reported that the incidence rate for radiation myelitis is 0.2 , $6 \%$ and $50 \%$ the total dose for $50 \mathrm{~Gy}, 60 \mathrm{~Gy}$, and 69 Gy respectively, when administrated at the conventional fraction of 2 Gy/day. In addition, according to the Radiation Oncology Group of European Organisation for Research and Treatment of Cancer, the maximal radiation dose that should be applied to the Spinal Cord is 45 Gy, and it should not exceed 40 Gy if oxaliplatin Chemotherapy is administered as well [23]. In this study the Spinal Cord dose did not reach the tolerance level in both techniques. Hovever, Sparing of spinal cord was significantly higher with Rapid Arc ( $p=0.047)$ than IMRT. Betzel et al. [24] reported that RapidArc deliveries were found to be more tolerant to variations in dose rate, gantry position, and MLC leaf position than fixed-field IMRT with dynamic SW delivery. Dose rate variations during RapidArc must be large to affect dose distributions significantly; dynamic IMRT is inherently not susceptible to such variations. Clinically acceptable treatments may be delivered accurately using Rapid Arc despite large fluctuations in dose rate and gantry position [24]. The dose to targets was delivered with more degrees of the freedom in Rapid Arc technique allowed to spare significantly the larynx $(p=0.002)$ and uninvolved oral cavity ( $p=0.004$ ) which are located in between the targets.

Concerning Healthy Tissue, the irradiation of higher volume by lower dose $(p=0.828)$ was not differ significantly. However, higher dose irradiation volume $\mathrm{V}_{30 \mathrm{~Gy}}$ was lesser with Rapid Arc significantly $(p=0.037)$. Computed integral dose of prescribed dose irradiation at non-tumor volume with Rapid Arc was lesser than with IMRT, there was no significant difference $(p=0.828)$. Jia.et al. found that the scatter dose predominates in the peripheral dose in VMAT, while the leakage dose predominates in IMRT, Moreover, leakage dose contribution to the peripheral dose becomes quite large and the peripheral dose at distant point is higher [25]. When evaluating radiation dose to healthy tissues, especially at sites remote from the treatment region, radiation leakage may represent an important factor, increasing integral dose to normal structures [26]. Specifically, two main sources of leakage from LINAC can be considered to increase the patient's Non-Tumour Integral Dose (NTID). (i) transmission of radiation through the collimator leaves and leakage through the primary collimation system. It is generally believed that leakage through the leaves of a conventional collimator might carry a significant contribution to peripheral integral dose (leakage is about 2.5\% for $6 \mathrm{MV}$ photon beams). (ii) leakage radiation is the dominant component and is directly related to the number of MU delivered [26]. Such a leakage radiation doses can increase the risk of secondary malignancy. In our study, the required monitor unit to deliver the prescribed dose with similar dose distribution of targets and OARs with IMRT was three times more than Rapid Arc ( $p<$ 0.00001). The impact of irradiation of healthy tissues at low doses with the VMAT technique is remains unre- 
solved. Longer treatment delivery times increase the chance of patient displacement during IMRT [27]-[29]. The treatment time for delivering the Rapid Arc plans was significantly lesser $(p<0.00001)$ than IMRT. This difference may have significantly impact on IMRT outcome, especially for tumors with a low $\alpha / \beta$ ratio and a short repair half time [30].

It is important to understand that whether treating with Rapid Arc or IMRT, pre treatment quality assurance is vital to safe and effective patient care. The reliability and efficacy of the Rapid Arc modality as anticipated in dosimetric investigation [31]-[33]. The results of our study was, the GAI scored with 3mm and 3\% constraints was more than acceptable threshold of $95 \%$ with both techniques. Our QA passing results agree with Verbakel et al. Published report [34]. High resolution of the detector (EPID) emphasizes the difficulties of the treatment planning system in properly managing the interleaf transmission and tongue and groove effect to notify the failing points in the delivery.

\section{Conclusion}

The Rapid Arc (VMAT) delivered the dose rapidly with lesser Monitor Unit without compromising in target dose coverage, conformity and higher sparing of OARs than IMRT. Thus, the risk of intra-fractional patient movement is reduced. The collimator transmission as scattered radiation from the Linac, Which is proportional to the number of $\mathrm{MU}$ and can increase potential risk of secondary cancer is now largely reduced by the Rapid Arc. These advantages can allow increase in dose/fraction and deliver in very short time a higher dose level at the same time keep maintain lesser OARs dose.

\section{References}

[1] Studenski, M.T., et al. (2013) Clinical Experience Transitioning from IMRT to VMAT for Head and Neck Cancer. Medical Dosimetry, 38, 171-175.

[2] Zhang, T., et al. (2015) Double-Arc Volumetric Modulated Therapy Improves Dose Distribution Compared to Static Gantry IMRT and 3D Conformal Radiotherapy for Adjuvant Therapy of Gastric Cancer. Radiation Oncology, 10, 114. http://dx.doi.org/10.1186/s13014-015-0420-X

[3] Kam, M.K., Chau, R.M., Suen, J., Choi, P.H. and Teo, P.M. (2003) Intensity-Modulated Radiotherapy in Nasopharyngeal Carcinoma: Dosimetric Advantage over Conventional Plans and Feasibility of Dose Escalation. International Journal of Radiation Oncology*Biology*Physics, 56, 145-157. http://dx.doi.org/10.1016/S0360-3016(03)00075-0

[4] Hummel, S., Simpson, E.L., Hemingway, P., Stevenson, M.D. and Rees, A. (2010) Intensity Modulated Radiotherapy for the Treatment of Prostate Cancer: A Systematic Review and Economic Evaluation. Health Technology Assessment, 14, 1-108, iii-iv. http://dx.doi.org/10.3310/hta14470

[5] Murshed, H., Liu, H.H., Liao, Z., Barker, J.L., Wang, X., Tucker, S.L., et al. (2004) Dose and Volume Reduction for Normal Lung Using Intensity-Modulated Radiotherapy for Advanced-Stage Non-Small-Cell Lung Cancer. International Journal of Radiation Oncology*Biology*Physics, 58, 1258-1267. http://dx.doi.org/10.1016/j.ijrobp.2003.09.086

[6] Otto, K. (2008) Volumetric Modulated Arc Therapy: IMRT in a Single Arc. Medical Physics, 35, 310-317. http://dx.doi.org/10.1118/1.2818738

[7] Johnston, M., Clifford, S., Bromley, R., et al. (2011) Volumetric-Modulated Arctherapy in Head and Neck Radiotherapy: A Planning Comparison Using Simultaneous Integrated Boost for Nasopharynx and Oropharynx Carcinoma. Clinical Oncology, 23, 503-511. http://dx.doi.org/10.1016/j.clon.2011.02.002

[8] Paddick, I. (2000) A Simple Scoring Ratio to Index the Conformity of Radiosurgical Treatment Plans. Technical note. Journal of Neurosurgery, 93, 219-222.

[9] Low, D.A., Harms, W.B., Mutic, S., et al. (1998) A Technique for Quantitative Evaluation of Dose Distribution. Medical Physics, 25, 656-661. http://dx.doi.org/10.1118/1.598248

[10] Nicolini, G., Fogliata, A., Vanetti, E., Clivio, A. and Cozzi, L. (2006) GLAaS: An Absolute Dose Calibration Algorithm for an Amorphous Silicon Portal Imager. Applications to IMRT Verifications. Medical Physics, 33, $2839-2851$. http://dx.doi.org/10.1118/1.2218314

[11] Nicolini, G., Vanetti, E., Clivio, A., Fogliata, A., Korreman, S., Bocanek, J. and Cozzi, L. (2008) The GLAaS Algorithm for Portal Dosimetry and Quality Assurance of RapidArc, an Intensity Modulated Rotational Therapy. Radiation Oncology, 3, 24. http://dx.doi.org/10.1186/1748-717X-3-24

[12] Cozzi, L., Dinshaw, K.A., Shrivastava, S.K., et al. (2008) A Treatment Planning Study Comparing Volumetric Arc Modulation with Rapid Arc and Fixed Field IMRT for Cervix Uteri Radiotherapy. Radiotherapy and Oncology, 89, 180-191. http://dx.doi.org/10.1016/j.radonc.2008.06.013 
[13] Palma, D., Vollans, E., James, K., et al. (2008) Volumetric Modulated Arc Therapy for Delivery of Prostate Radiotherapy: Comparison with Intensity-Modulated Radiotherapy and Three-Dimensional Conformal Radiotherapy. International Journal of Radiation Oncology*Biology*Physics, 72, 996-1001. http://dx.doi.org/10.1016/j.ijrobp.2008.02.047

[14] Clivio, A., Fogliata, A., Franzetti-Pallanda, A., et al. (2009) Volumetric-Modulated Arc Radiotherapy for Carcinomas of the Anal Canal: A Treatment Planning Comparison with Fixed Field IMRT. Radiotherapy and Oncology, 92, 118124. http://dx.doi.org/10.1016/j.radonc.2008.12.020

[15] Wagner, D., Christiansen, H., Wolff, H. and Vorwerk, H. (2009) Radiotherapy of Malignant Gliomas: Comparison of Volumetric Single Arc Technique and 3D Conformal Technique. Radiotherapy and Oncology, 93, 593-596. http://dx.doi.org/10.1016/j.radonc.2009.10.002

[16] Mellon, E.A., Javedan, K., Strom, T.J., et al. (2015) A Dosimetric Comparison of Volumetric Modulated Arc Therapy with Step-and-Shoot Intensity Modulated Radiation Therapy for Prostate Cancer. Practical Radiation Oncology, 5, 1115. http://dx.doi.org/10.1016/j.prro.2014.03.003

[17] Mahantshetty, U., Jamema, S., Engineer, R., et al. (2010) Whole Abdomen Radiation Therapy in Ovarian Cancers: A Comparison between Fixed Beam and Volumetric Arc Based Intensity Modulation. Radiation Oncology, 5, 106. http://dx.doi.org/10.1186/1748-717X-5-106

[18] Braam, P.M., Terhaard, C.H., Roesink, J.M. and Raaijmakers, C.P. (2006) Intensity-Modulated Radiotherapy Significantly Reduces Xerostomia Compared with Conventional Radiotherapy. International Journal of Radiation Oncology*Biology*Physics, 66, 975-980. http://dx.doi.org/10.1016/j.ijrobp.2006.06.045

[19] Chao, K.S., Majhail, N., Huang, C.J., Simpson, J.R., Perez, C.A., Haughey, B. and Spector, G. (2001) Intensity-Modulated Radiation Therapy Reduces Late Salivary Toxicity without Compromising Tumor Control in Patients with Oropharyngeal Carcinoma: A Comparison with Conventional Techniques. Radiotherapy and Oncology, 61, 275-280. http://dx.doi.org/10.1016/S0167-8140(01)00449-2

[20] Dijkema, T., Terhaard, C.H., Roesink, J.M., Braam, P.M., van Gils, C.H., Moerland, M.A. and Raaijmakers, C.P. (2008) Large Cohort Dose-Volume Response Analysis of Parotid Gland Function after Radiotherapy: Intensity-Modulated versus Conventional Radiotherapy. International Journal of Radiation Oncology*Biology*Physics, 72, 1101-1109. http://dx.doi.org/10.1016/j.ijrobp.2008.02.059

[21] Braam, P.M., Roesink, J.M., Moerland, M.A., Raaijmakers, C.P., Schipper, M. and Terhaard, C.H. (2005) Long-Term Parotid Gland Function after Radiotherapy. International Journal of Radiation Oncology*Biology*Physics, 62, 659664. http://dx.doi.org/10.1016/j.ijrobp.2004.12.015

[22] Kirkpatrick, J.P., van der Kogel, A.J. and Schultheiss, T.E. (2010) Radiation Dose-Volume Effects in the Spinal Cord. International Journal of Radiation Oncology*Biology*Physics, 76, S42-S49. http://dx.doi.org/10.1016/j.ijrobp.2009.04.095

[23] Matzinger, O., Gerber, E., Bernstein, Z., Maingon, P., Haustermans, K., Bosset, J.F., et al. (2009) EORTC-ROG Expert Opinion: Radiotherapy Volume and Treatment Guidelines for Neoadjuvant Radiation of Adenocarcinomas of the Gastroesophageal Junction and the Stomach. Radiotherapy and Oncology, 92, 164-175. http://dx.doi.org/10.1016/j.radonc.2009.03.018

[24] Betzel, G.T., Yi, B.Y., Niu, Y. and Yu, C.X. (2012) Is RapidArc More Susceptible to Delivery Uncertainties than Dynamic IMRT? Medical Physics, 39, 5882-5890. http://dx.doi.org/10.1118/1.4749965

[25] Jia, M.X., Zhang, X., Yin, C., et al. (2014) Peripheral Dose Measurements in Cervical Cancer Radiotherapy: A Comparison of Volumetric Modulated Arc Therapy and Step-and-Shoot IMRT Techniques. Radiotherapy and Oncology, 9, 61. http://dx.doi.org/10.1186/1748-717X-9-61

[26] Petti, P.L., Chuang, C.F., Smith, V. and Larson, D.A. (2006) Peripheral Doses in Cyber Knife Radiosurgery. Medical Physics, 33, 1770-1779. http://dx.doi.org/10.1118/1.2198173

[27] Verbakel, W.F.A.R., Cuijpers, J.P., Hoffmans, D., et al. (2009) Volumetric Intensity-Modulated Arc Therapy vs. Conventional IMRT in Head-and-Neck Cancer: A Comparative Planning and Dosimetric Study. International Journal of Radiation Oncology*Biology*Physics, 74, 252-259. http://dx.doi.org/10.1016/j.ijrobp.2008.12.033

[28] Bertelsen, A., Hansen, C.R., Johansen, J., et al. (2010) Single Arc Volumetric Modulated Arc Therapy of Head and Neck Cancer. Radiotherapy and Oncology, 95, 142-148. http://dx.doi.org/10.1016/j.radonc.2010.01.011

[29] Kim, S., Akpati, H.C., Kielbasa, J.E., et al. (2004) Evaluation of Intrafraction Patient Movement for CNS and Head \& Neck IMRT. Medical Physics, 31, 500-506. http://dx.doi.org/10.1118/1.1644641

[30] Wang, J.Z., Li, X.A., D’Souza, W.D. and Stewart, R.D. (2003) Impact of Prolonged Fraction Delivery Times on Tumor Control: A Note of Caution for Intensity-Modulated Radiation Therapy (IMRT). International Journal of Radiation Oncology*Biology*Physics, 57, 543-552. http://dx.doi.org/10.1016/S0360-3016(03)00499-1

[31] Verbakel, W., Cuijpers, J., Hoffmans, D., Bieker, M. and Slotman, B. (2009) Volumetric Intensity Modulated Arc Therapy versus Conventional IMRT in Head and Neck Cancer: A Comparative Planning and Dosimetric Study. Inter- 
national Journal of Radiation Oncology*Biology*Physics, 74, 252-259. http://dx.doi.org/10.1016/j.ijrobp.2008.12.033

[32] Vanetti, E., Clivio, A., Nicolini, G., Fogliata, A., Ghosh-Laskar, S., Agarwal, J.P., Upreti, R.R., Budrukkar, A., Murthy, V., Deshpande, D.D., Shrivastava, S.K., Dinshaw, K.A. and Cozzi, L. (2009) Volumetric Modulated Arc Radiotherapy for Carcinomas of the Oro-Pharynx, Hypo-Pharynx and Larynx: A Treatment Planning Comparison with Fixed Field IMRT. Radiotherapy and Oncology, 92, 111-117. http://dx.doi.org/10.1016/j.radonc.2008.12.008

[33] Doornaert, P., Verbakel, W., Bieker, M., Slotman, B.J. and Senan, S. (2011) RapidArc Planning and Delivery in Patients with Locally Advanced Head-and-Neck Cancer Undergoing Chemoradiotherapy. International Journal of Radiation Oncology*Biology*Physics, 79, 429-435. http://dx.doi.org/10.1016/j.ijrobp.2009.11.014

[34] Verbakel, W., Cuijpers, J., Hoffmans, D., Bieker, M. and Slotman, B. (2009) Volumetric Intensity Modulated Arc Therapy versus Conventional IMRT in Head and Neck Cancer: A Comparative Planning and Dosimetric Study. International Journal of Radiation Oncology*Biology*Physics, 74, 252-259. http://dx.doi.org/10.1016/j.jjrobp.2008.12.033 